\title{
Participación de la mujer en la estructura organizativa de la SAT Guatemala
}

\section{Participation of women in the organizational structure of SAT Guatemala}

\section{Referencia del artículo}

\author{
- Diana Sofía Felícita Orenos Pineda y Elba Marina \\ - Monzón Dávila \\ Universidad de San Carlos de Guatemala \\ dianitaorenos@gmail.com \\ https://orcid.org/0000-0003-3636-1265
}

Orenos, D. y Monzón, E. (2020). Participación de la mujer en la estructura organizativa de la SAT, Guatemala. Revista Guatemalteca de Educación Superior, 3(1), 74-84.

DOI: https://doi.org/10.46954/revistages.v111.6

Recibido 20/05/2020.

Revisado 21/05/2020.

Aceptado 20/06/2020.

Palabras clave

mujer, participación, estructura organizativa

\section{Resumen}

La investigación fue orientada a los estudios de género dentro de la Superintendencia de Administración Tributaria -SAT-, institución descentralizada del Estado de Guatemala. Se empleó metodología cuantitativa, de alcance descriptivo. Se respondió la pregunta ¿Cómo se evidencia la participación de la mujer dentro de la estructura organizacional de la Superintendencia de Administración Tributaria? Para efectos de la investigación se tomó como población y muestra a todo el personal de la SAT, puesto que fueron analizadas las bases de datos de la nómina institucional, documentos institucionales, el diagnóstico de género realizado por la (USAID, 2018), entre otros documentos de interés y significancia. Esta institución ha tenido un incremento del $2 \%$ en la participación femenina dentro de su estructura organizacional a partir del año 2017. 


\section{Abstract}

The research was oriented to gender studies within the Superintendence of Tax Administration -SAT-, a decentralized institution of the State of Guatemala. A quantitative methodology was used, with a descriptive scope. The question was answered: How is women's participation evidenced within the organizational structure of the Superintendence of Tax Administration? For the purposes of the research, the population and sample were all SAT personnel, since the institutional payroll databases, institutional documents, the gender diagnosis conducted by USAID (2018), among other documents of interest and significance, were analyzed. This institution has had a $2 \%$ increase in female participation within its organizational structure since 2017.

\section{Introducción}

La participación de la mujer en el contexto social y político ha ido incrementando en las últimas décadas, esto se debe a que la mujer cada día toma más fuerza para presentarse y desempeñar un papel protagónico en áreas poco exploradas para las féminas. La Organización de las Naciones Unidas, Organización de Estados Americanos, entre otras entidades internacionales con influencia global, han sentado las bases para que cada vez más mujeres se adentren en contextos y labores antes denominadas "trabajo de hombre".

Todo esto ha permitido que cada vez más los estudios de género cobren importancia y es por ello que la investigación que se presenta a continuación cobra relevancia y pertinencia en el contexto guatemalteco. Existen pocos estudios relacionados con la participación de la mujer en entidades descentralizadas del Estado de Guatemala.

\section{Materiales y métodos}

El enfoque metodológico de esta investigación es cuantitativo puesto que se realizó la descripción de datos, dando como resultado números, porcentajes y medidas. Posee un alcance descriptivo.

\section{Keywords}

women, participation, organizational structure, gender 
Las variables y unidades de medida empleadas corresponden a la estructura organizacional de la Superintendencia de Administración Tributaria y la participación de la mujer, busca determinar la estructura organizacional de la Superintendencia de Administración Tributaria, pero en torno a la participación de la mujer, la línea de investigación dirige a la importancia del estudio de género, en este caso particular, el género femenino. Para la realización de esta investigación se ha tomado como población el cien por ciento del personal de la SAT. Se analizó la información de todos los elementos de la población con la finalidad de generar resultados con un $99 \%$ de confianza.

La recolección de datos se llevó a cabo por medio de una solicitud oficial basada en el Decreto 57-2008, Ley de Acceso a la Información Pública. Esto permitió que los datos recabados fueran analizados a partir de la tabulación e interpretación de estos por medio del Programa Microsoft Excel, analizando las unidades de medida previamente establecidas. Este software contiene las características que lo hacen confiable y adecuado para el análisis de la información.

\section{Resultados}

\section{Descripción y datos históricos de la investigación}

En el año 2017, la Superintendencia de Administración Tributaria -SAT- participó en el primer análisis de género del sistema tributario guatemalteco, el cual generó información relevante y posteriormente utilizada por USAID. En el año 2018 USAID realizó una publicación denominada Diagnóstico de Tributación y Género en Guatemala. Dentro de este documento se dieron a conocer datos que fueron analizados en relación con la forma en la que funciona el sistema tributario guatemalteco. Dentro de los contenidos de esta publicación, USAID afirmó que aquel diagnóstico realizado por la SAT permitió que se observara "la ausencia de la perspectiva de género en documentos sustantivos, que reflejen cambios a lo interno y externo de la institución, lo que reproduce desigualdades". (USAID F. t., 2018). 
Figura 1. Trabajadores de la SAT

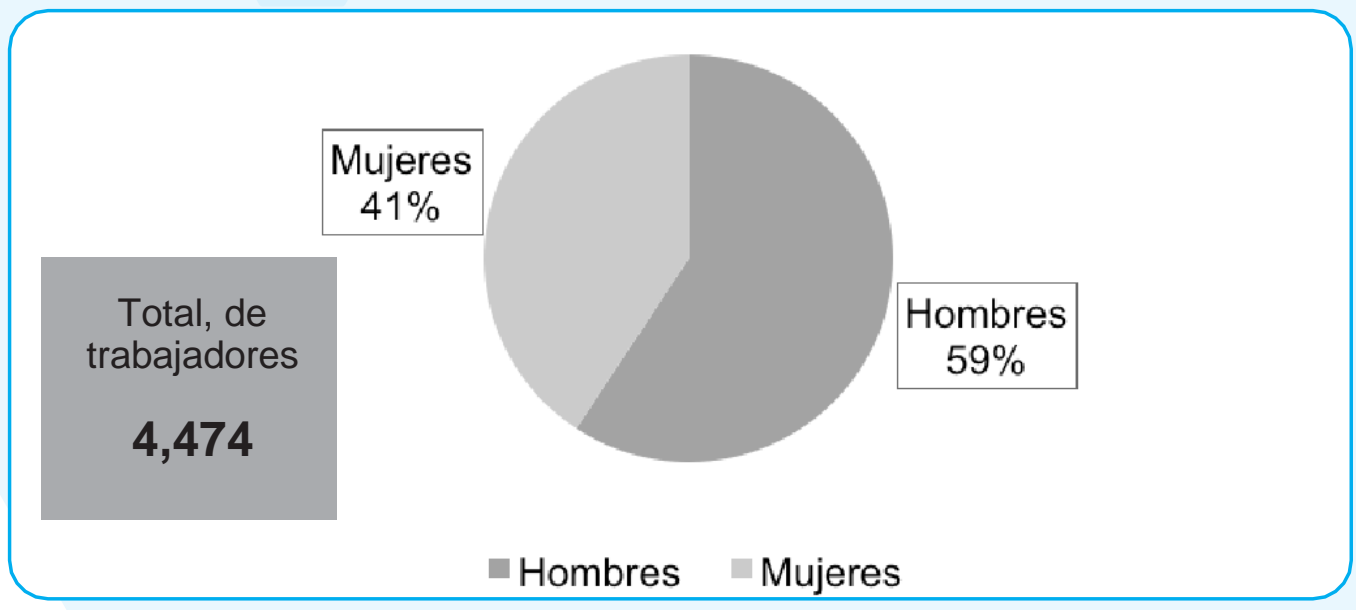

Fuente: Orenos (2020).

La Superintendencia de Administración Tributaria presentaba un $41 \%$ de mujeres y un $59 \%$ de hombres laborando, lo cual sumaba un total aproximado de 4,474 trabajadores, esto muestra una brecha del $18 \%$ entre ambos sexos y un $9 \%$ para llegar a la equidad. Datos según Diagnóstico Institucional de Género (2017), citado en (USAID F. t., 2018).

Figura 2. Puestos de decisión ocupados por género

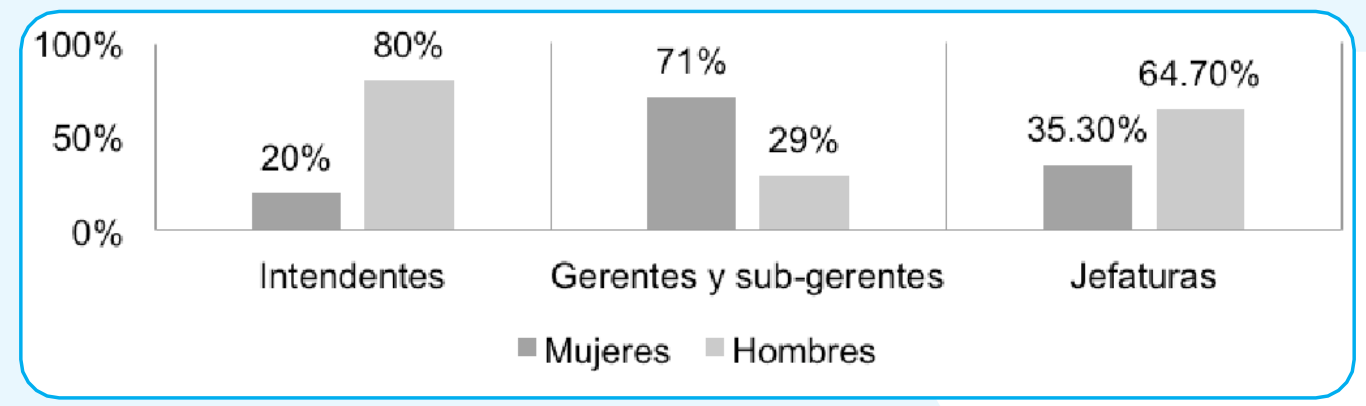

Fuente: Orenos (2020).

La figura muestra los porcentajes de hombres y mujeres trabajadores en la SAT para el 2017, es posible apreciar que para 2018 los porcentajes de mujeres eran menores en puestos de decisión correspondientes a Intendencias y jefaturas, esto según el Diagnóstico Institucional de Género (2017), citado en (USAID F. t., 2018). 
Figura 3. Población por género

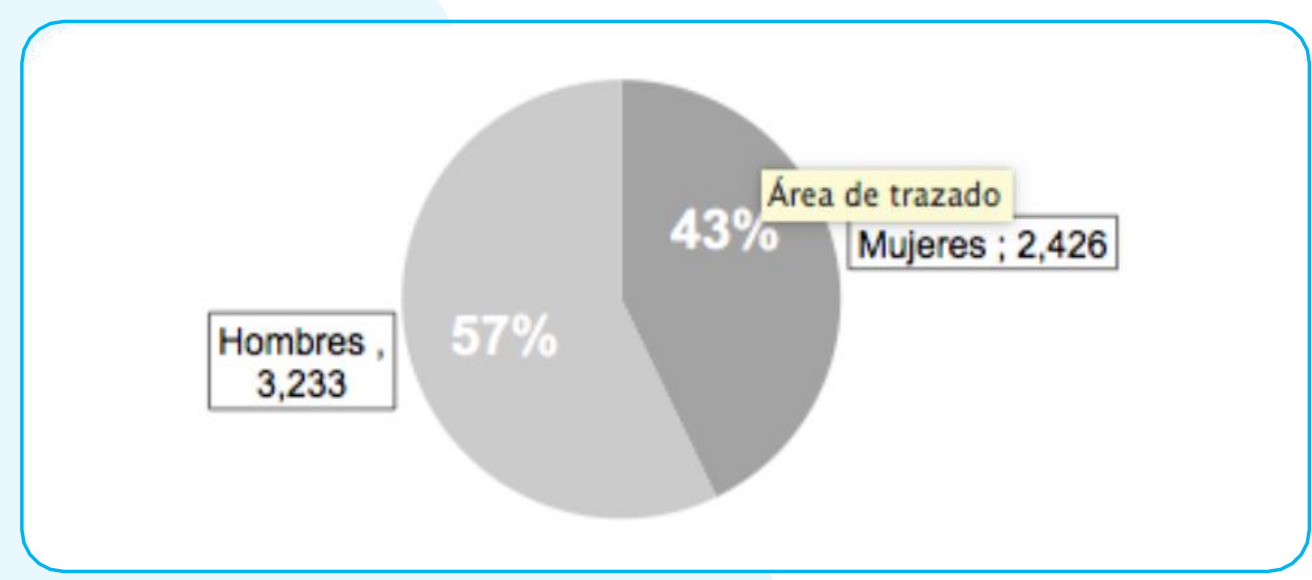

Fuente: Orenos (2020).

En la figura es posible apreciar de manera clara la proporción de hombres y mujeres que tenía la SAT para marzo de 2020. En comparación con los datos anteriores, hay incremento en el porcentaje de mujeres lo cual establece una brecha de igualdad de aproximadamente $7 \%$ y un incremento del $2 \%$ con respecto a 2017.

Figura 4. Población por renglón presupuestario

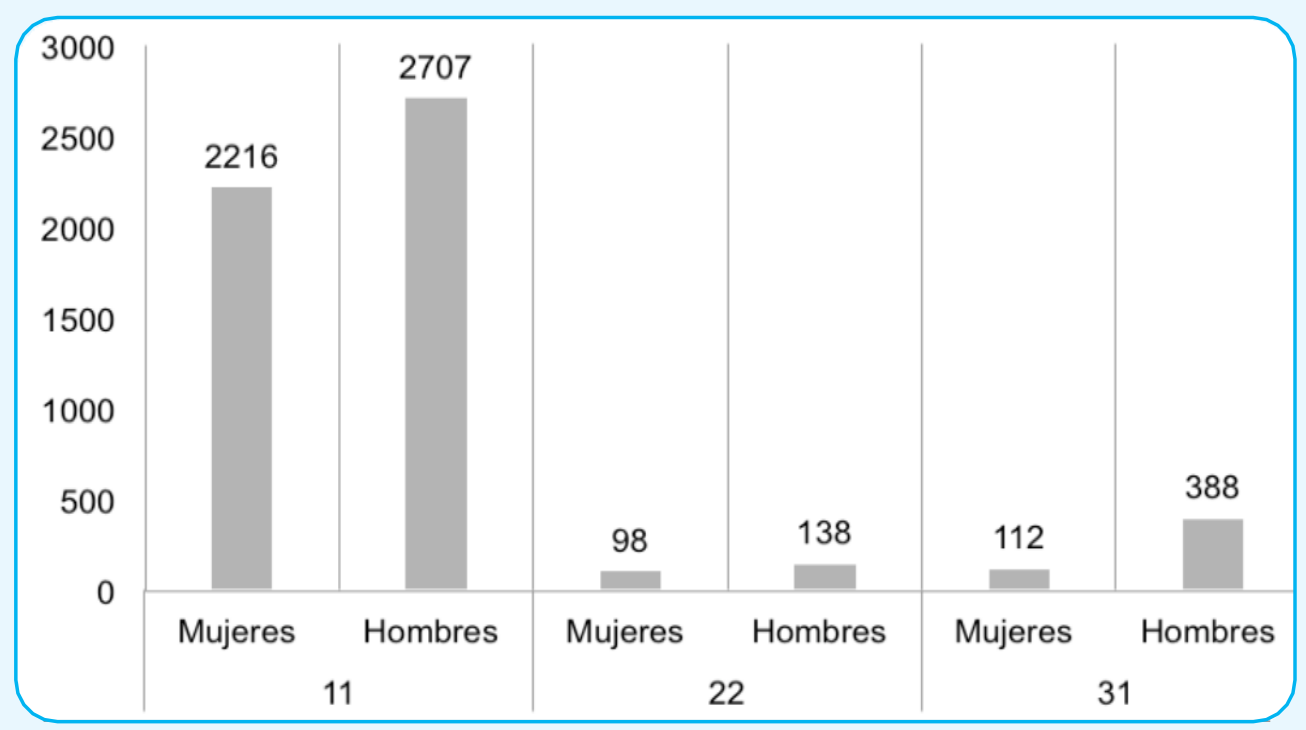

Fuente: Orenos (2020).

La figura permite apreciar desde una perspectiva amplia, cuál es la realidad institucional en cuanto a la proporción de hombres y mujeres por renglón presupuestario, es importante apreciar que 
dentro de las personas contratadas en los renglones 0-11, 0-22 y 0-31 el número de mujeres siempre es menor que el número de hombres. En el renglón 011, la diferencia entre ambos es de aproximadamente de 491 hombres más que mujeres.

Figura 5. Población por denominación

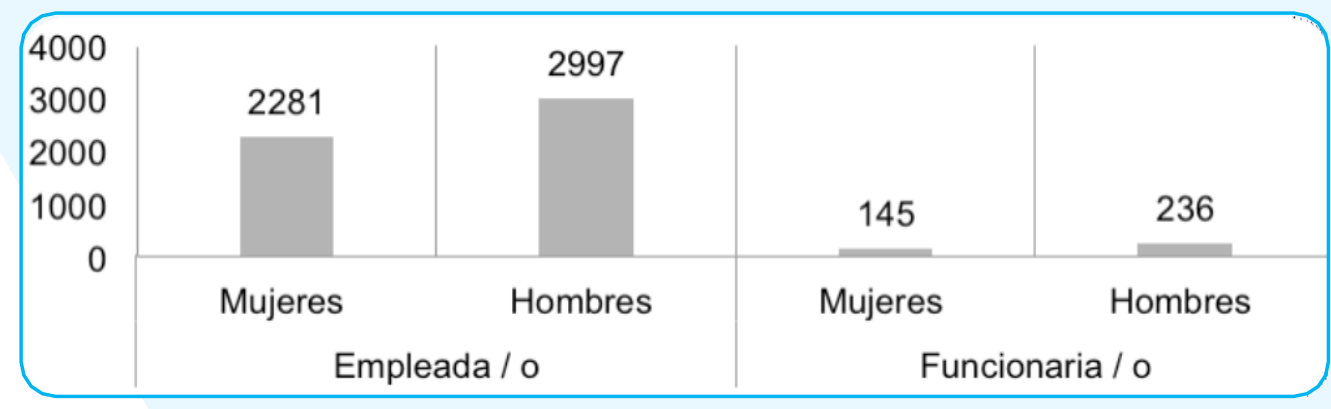

Fuente: Orenos (2020).

La figura anterior muestra la clasificación de hombres y mujeres por denominación empleado o funcionario, según clasifica la SAT a su personal. Se aprecia que las mujeres tienen menor participación en ambas denominaciones, siendo la que posee mayor brecha la denominación "funcionario" donde vemos que de 381 personas, únicamente 145 son mujeres, para marzo de 2020. En cuanto a la denominación "empleado" tiene una diferencia de 716 hombres.

Figura 6. Población por categoría ocupacional

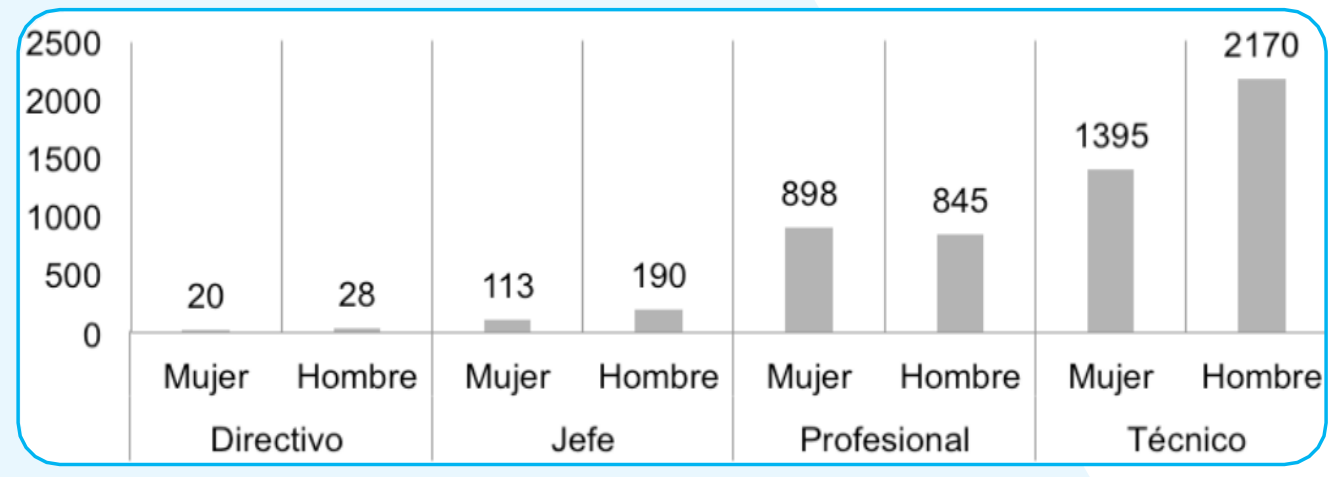

Fuente: Orenos (2020).

La figura anterior muestra el número de hombres y mujeres que laboran en la SAT, clasificado por las categorías ocupacionales 
establecidas para efectos de esta investigación. Se puede apreciar que la única categoría ocupacional que tiene mayor número de mujeres laborando, según la nómina es la de "Profesional", en ella es posible apreciar una diferencia de 53 personas. En cuanto a las otras categorías ocupacionales la proporción de mujeres es menor, siendo la más evidente la de "técnico" donde se aprecia una diferencia de 775 personas.

Figura 7. Escalas salariales por género

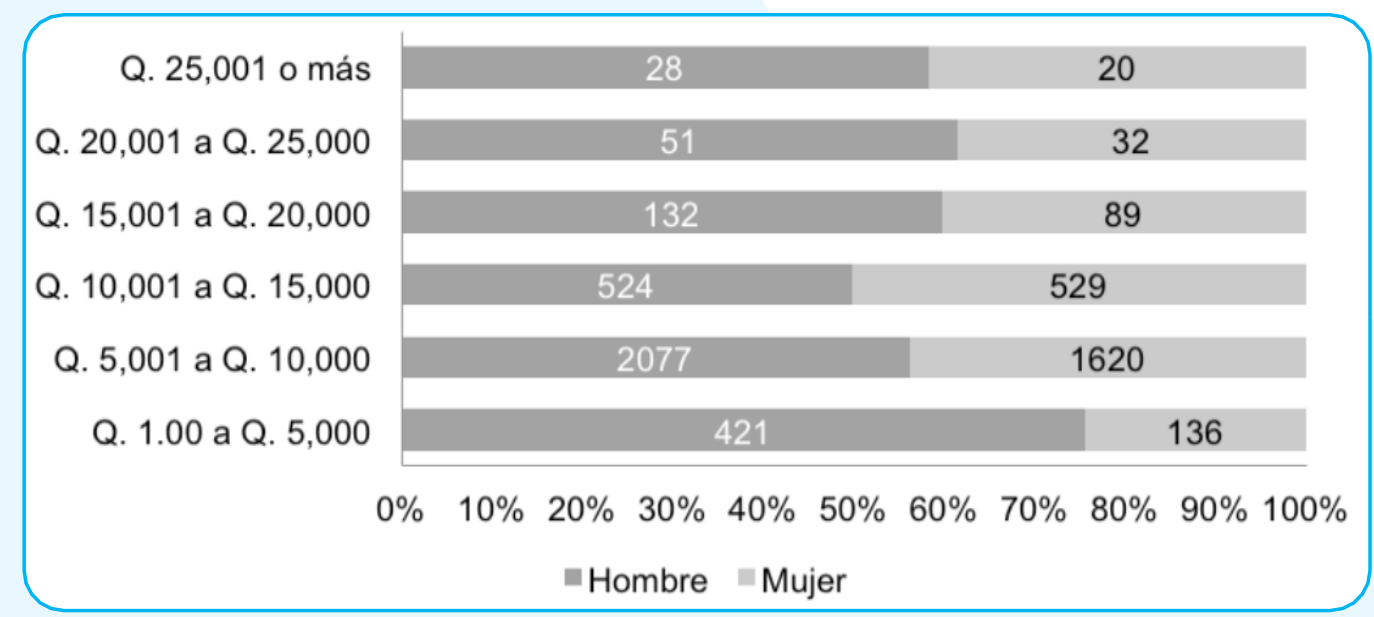

Fuente: Orenos (2020).

Esta figura muestra de manera detallada las escalas salariales realizadas para efectos de esta investigación, se aprecia que la única escala salarial que tiene mayor cantidad de mujeres es la correspondiente a Q. 10,001.00 a Q15,000.00 en la que puede apreciarse una diferencia de únicamente cinco personas. La escala más alarmante por su diferencia es la correspondiente a Q. 1.00 a Q. 5,000 la cual se aprecia una diferencia de 285 hombres más que mujeres. Estos datos proporcionan un escenario general en cuanto a escalas salariales.

Tabla 1. Brecha salarial entre hombres y mujeres, sumatoria salarial

\begin{tabular}{ccccc}
\hline Género & $\begin{array}{c}\text { Hombres } \\
\text { (En Q.) }\end{array}$ & $\begin{array}{c}\text { Mujeres } \\
\text { (En Q.) }\end{array}$ & Total & Brecha salarial \\
\hline Sumatoria & $26,615,202.92$ & $21,256,227.60$ & $47,871,430.52$ & $5,358,975.32$ \\
\hline
\end{tabular}


En la tabla anterior, se evidencia que la sumatoria de salarios de hombres es superior a la sumatoria de salarios de las mujeres en cinco millones trescientos cincuenta y ocho mil novecientos setenta y cinco quetzales con treinta y dos centavos, esto corresponde a una diferencia del $11.19 \%$ de la sumatoria de salarios de la nómina de trabajadores de la Superintendencia de Administración Tributaria -SAT-. Lo interesante de este análisis es que, para marzo de 2020, la Superintendencia de Administración Tributaria poseía una brecha porcentual de hombres y mujeres correspondientes al 14\% y una brecha salarial de Q. 5,358,975.32. Ambos datos debieran reducirse en los próximos años de manera proporcional para poder contribuir a la igualdad de género en la estructura organizacional de la SAT, en Guatemala.

\section{Medidas de tendencia central por salario}

Según resultados de esta investigación (2020), diseño elaboración propia, (2020) la media de salarios de mujeres trabajadoras de la SAT es de Q. 8,762.84, mientras que la media del salario de hombres es de Q. 8,232.35; esto quiere decir que el salario promedio de las trabajadoras de la SAT es Q. 529.49 mayor al salario promedio de los trabajadores de esta institución.

Asimismo, es posible apreciar que la cifra de la mediana y la moda en salarios, tanto de hombres como de mujeres es de Q.6,961.00. Este mismo análisis arrojó que el rango de los salarios obtenidos por las mujeres en la SAT corresponde a una diferencia de Q. 61,370.00. Esto quiere decir que la diferencia en quetzales existente entre el salario de la mujer que menos gana en la SAT y la mujer que más gana, corresponde a esta cantidad salarial neta.

Por otro lado, según los datos analizados, es posible apreciar que el rango de los salarios percibidos por los hombres es de Q.45, 720.00, sin embargo, es importante aclarar que en el momento de recibir los datos utilizados para esta investigación, la Superintendencia de Administración Tributaria -SAT- poseía un superintendente interino, por lo que no figuró en nómina la figura del superintendente. Pese a esta circunstancia mencionada en el 
párrafo anterior, se realizó una simulación de datos, incluyendo este reajuste salarial que no estaba incluido entre las cifras y la única variable que se obtuvo fue en el rango salarial de los hombres.

\section{Discusión}

La investigación realizada en la institución refleja inclusión de las mujeres, sin embargo, cuando se hizo el análisis de las nóminas y bases de datos la evaluación reflejó que hay un porcentaje de disparidad muy significativo, por lo que la evaluación efectuada determinó que aún no existe equidad de género en la estructura organizativa y por ende no se logra contribuir al fortalecimiento de la gestión institucional en un alto porcentaje. Dentro de la estructura organizacional de la Superintendencia de Administración Tributaria -SAT- fue posible apreciar que existe participación de la mujer, ya que de las veintiséis dependencias que la conforman, diez tienen mayor proporción y siete son lideradas por mujeres. Por lo que es imperativo buscar maneras de abrir espacios y rutas para que la mujer pueda optar a este tipo de puestos y ser electa para ellos.

Se determinó que la institución posee una nómina de 5,659 personas que laboran en las dependencias que la conforman, sin embargo, solo el $47 \%$ son mujeres y $53 \%$ son hombres. Aunque el porcentaje de hombres y mujeres no es equitativo, se encontró información de que la cifra de mujeres dentro de esta institución ha aumentado en un $2 \%$ desde el diagnóstico de género realizado durante el año 2017.

La representación de las mujeres en la Superintendencia de Administración Tributaria -SAT- evidencia que en el periodo del año 2019 a marzo 2020 hay un $47 \%$ de ellas laborando en todas sus dependencias. Sin embargo, a pesar de haber mujeres en puestos de decisión y representación con cargos fijos dentro del Directorio, no todas las dependencias son lideradas por mujeres, ya que los resultados de la investigación arrojaron que 145 mujeres tienen funciones de jefaturas, directivas o liderazgo administrativo y 236 hombres tienen cargos similares, evidenciando que en la actualidad el papel de la mujer es inferior a la participación del hombre. 


\section{Agradecimientos}

A la Superintendencia de Administración Tributaria -SAT- y su Unidad de Información Pública por la disponibilidad en cuanto a proporcionar los documentos, bases de datos y demás información necesaria para la realización de esta investigación. A la Doctora María lliana Cardona Monroy por compartir sus conocimientos y la asesoría constante. A la Doctora Elba Marina Monzón Dávila de Barillas por el apoyo brindado en cuanto al asesoramiento para la realización de esta investigación.

\section{Referencias}

CEPAL, D. d. (2014). Informe de Estado sobre la declaración y la plataforma de Beijing y los resultados del XXIII período de sesiones de la Asamblea General de la ONU. Guatemala: División de Asuntos de Género de la CEPAL.

Gobierno de Guatemala, G. d. (2014). Informe de Estado sobre la Declaración y la Plataforma de Acción de Beijing y los Resultados del XXIII Período de Sesiones de la Asamblea General de la ONU. Guatemala: Gobierno de Guatemala.

Guatemala, O. M. (2017). Reporte Anual 2017. Guatemala: ONU Mujeres.

Mujer, S. P. (2018). Estrategia de seguimiento a la convención sobre la eliminación de todas las formas de discriminación contra la mujer. Guatemala: SEPREM.

Naciones Unidas, N. U. (2014). Los Derechos de las Mujeres también son Derechos Humanos. Nueva York y Ginebra: Naciones Unida, Oficina del Alto Comisionado.

PNUD, P. d. (2019). Programa de las Naciones Unidas para el Desarrollo. Recuperado el 18 de 11 de 2019, de https:// www.undp.org/content/undp/es/home/sdgoverview/mdg_ goals.html

Sánchez, F. M. (2009). La participación política de las mujeres en gobiernos locales latinoamericanos: barreras y desafíos para una efectiva democracia de género. Barcelona.

SEGEPLAN, S. y. (2018). Lineamientos para incorporar el enfoque de equidad entre hombres y mujeres en la gestión integral de riesgo. Guatemala: SEPREM. 
SEPREM. (2018). Marco normativo y de política pública para el avance de las mujeres. Guatemala: SEPREM.

SEPREM, S. P. (2019). Agenda Estratégica. Guatemala.

SEPREM, S. P. (2019). Informe de Avances de Clasificador de Presupuesto con Enfoque de Género. Guatemala: SEPREM.

UNESCO. (2016). Guía para la igualdad de género en las políticas y prácticas de la formación docente. UNESCO.

UNESCO. (2016). Igualdad de Género. UNESCO.

USAID. (2018). Proyecto de Reforma Fiscal y Compras de Guatemala: Estudio de Análisis de Impacto Tributario con Enfoque a Género e Inclusión Social. Guatemala: USAID.

USAID, F. t. (2018). Diagnóstico de Tributación y Género en Guatemala. Guatemala: USAID.

\section{Sobre la autora}

Diana Sofía Felícita Orenos Pineda es Profesora de Enseñanza Media en Pedagogía y Técnico en Administración Educativa por la Facultad de Humanidades de la USAC. Licenciada en Pedagogía y Administración Educativa. Investigadora educativa de la Gerencia de Formación de Personal. Estudiante de la carrera de Maestría en Investigación, Escuela de Estudios de Posgrado, Facultad de Humanidades.

\section{Copyright (c) Diana Sofía Felícita Orenos Pineda y Elba Marina}

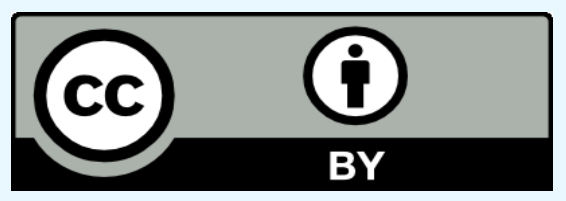

Este texto está protegido por una licencia CreativeCommons 4.0.

Usted es libre para compartir, copiar y redistribuir el material en cualquier medio o formato y adaptar el documento, remezclar, transformar y crear a partir del material para cualquier propósito, incluso comercialmente, siempre que cumpla la condición de atribución: usted debe reconocer el crédito de una obra de manera adecuada, proporcionar un enlace a la licencia, e indicar si se han realizado cambios. Puede hacerlo en cualquier forma razonable, pero no de forma tal que sugiera que tiene el apoyo del licenciante o lo recibe por el uso que hace. 